\title{
The KT-BRST Complex of a Degenerate Lagrangian System
}

\author{
D. BASHKIROV ${ }^{1}$, G. GIACHETTA ${ }^{2}$, L. MANGIAROTTI ${ }^{2}$ AND \\ G.SARDANASHVILY ${ }^{1}$ \\ ${ }^{1}$ Department of Theoretical Physics, Moscow State University, 117234 Moscow, Russia \\ ${ }^{2}$ Department of Mathematics and Informatics, University of Camerino, 62032 Camerino \\ $(M C)$, Italy
}

\begin{abstract}
Quantization of a Lagrangian field system essentially depends on its degeneracy and implies its BRST extension defined by sets of non-trivial Noether and higher-stage Noether identities. However, one meets a problem how to select trivial and non-trivial higherstage Noether identities. We show that, under certain conditions, one can associate to a degenerate Lagrangian $L$ the KT-BRST complex of fields, antifields and ghosts whose boundary and coboundary operators provide all non-trivial Noether identities and gauge symmetries of $L$. In this case, $L$ can be extended to a proper solution of the master equation. 70S05, 70S20
\end{abstract}

\section{Introduction}

The BV quantization of Lagrangian field system essentially depends on its degeneracy and implies its BRST extension given by the Koszul-Tate (henceforth KT) and BRST complexes [1, 5, 6, 9]. These complexes are defined by sets of non-trivial Noether identities (henceforth NI) and higher-stage NI. Any Euler-Lagrange operator satisfies NI which are separated into the trivial and non-trivial ones. These NI obey first-stage NI, which in turn are subject to the second-stage NI, and so on. One however meets a problem how to select trivial and non-trivial higher-stage NI.

Note that the notion of higher-stage NI has come from that of reducible constraints. The KT complex of NI has been invented similarly to that of constraints under the condition that NI are locally separated into independent and dependent ones [1, 6]. This condition is relevant for constraints, defined by a finite set of functions which the inverse mapping theorem is applied to. However, NI unlike constraints are differential equations. They are given by an infinite set of functions on a Fréchet manifold of infinite order jets where the inverse mapping theorem fails to be valid.

We consider a generic Lagrangian theory of even and odd variables on an $n$ dimensional smooth real manifold $X$. It is described in terms of the Grassmanngraded variational bicomplex [1, 3, 4, 8]. Accordingly, NI are represented by onecycles of a certain chain complex. Its boundaries are necessarily NI, called trivial. Non-trivial NI modulo the trivial ones are given by first homology of this complex. To describe $(k+1)$-stage NI, let us assume that non-trivial $k$-stage $\mathrm{NI}$ are generated by a projective $C^{\infty}(X)$-module $\mathscr{C}_{(k)}$ of finite rank and that a certain homology condition (Definition 1) holds [4]. In this case, $(k+1)$-stage NI are represented by $(k+2)$-cycles of some chain complex of modules of antifields isomorphic to $\mathscr{C}_{(i)}$, 
$i \leq k$. Accordingly, trivial $(k+1)$-stage NI are defined as its boundaries. Iterating the arguments, we come to the exact KT complex (16) with the boundary KT operator (14) whose nilpotentness is equivalent to all non-trivial NI (Theorem 2) [4].

The inverse second Noether theorem (Theorem 3) that we prove associates to the KT complex (16) the cochain sequence (20) the $\left(\wedge^{n} T^{*} X\right)$-duals of the modules $\mathscr{C}_{(k)}$ whose elements are called ghosts. Components of its ascent operator (21), called the gauge operator, are gauge and higher-stage gauge symmetries of an original Lagrangian $L$. The gauge operator need not be nilpotent. We show that, if it admits a nilpotent extension (41), an original Lagrangian $L$ is extended to a proper solution of the master equation (36), which the BV quantization starts with. In this case, the KT complex (16) and the cochain sequence (20) are combined into the KT-BRST complex which is a desired BRST extension of an original Lagrangian field theory.

\section{Lagrangian theory of even and odd fields}

Let us consider a composite bundle $F \rightarrow Y \rightarrow X$ where $F \rightarrow Y$ is a vector bundle provided with bundle coordinates $\left(x^{\lambda}, y^{i}, q^{a}\right)$. Jet manifolds $J^{r} F$ of $F \rightarrow X$ are also vector bundles $J^{r} F \rightarrow J^{r} Y$ coordinated by $\left(x^{\lambda}, y_{\Lambda}^{i}, q_{\Lambda}^{a}\right), 0 \leq|\Lambda| \leq r$, with respect to linear frames $\left\{e_{a}^{\Lambda}\right\}$, where $\Lambda=\left(\lambda_{1} \ldots \lambda_{k}\right),|\Lambda|=k$, denote symmetric multi-indices. Let $\left(J^{r} Y, \mathscr{A}_{r}\right)$ be a graded manifold whose body is $J^{r} Y$ and whose $C^{\infty}\left(J^{r} Y\right)$-ring of graded functions $\mathscr{A}_{r}$ is generated by sections of the dual $\left(J^{r} F\right)^{*}$ of $J^{r} F \rightarrow J^{r} Y$, i.e, it is locally generated by the coframes $\left\{c_{\Lambda}^{a}\right\}$ dual of the frames $\left\{e_{a}^{\Lambda}\right\}$. Let $\mathscr{S}_{r}^{*}[F ; Y]$ be the differential graded algebra (henceforth DGA) of graded differential forms on the graded manifold $\left(J^{r} Y, \mathscr{A}_{r}\right)$. There is the inverse system of jet manifolds $J^{r-1} Y \leftarrow$ $J^{r} Y$. Its projective limit is a Fréchet manifold coordinated by $\left(x^{\lambda}, y_{\Lambda}^{i}\right), 0 \leq|\Lambda|$. This inverse system yields the direct system of DGAs

$$
\mathscr{S}^{*}[F ; Y] \longrightarrow \mathscr{S}_{1}^{*}[F ; Y] \longrightarrow \cdots \mathscr{S}_{r}^{*}[F ; Y] \longrightarrow \cdots .
$$

Its direct limit $\mathscr{S}_{\infty}^{*}[F ; Y]$ is the DGA of all graded differential forms on graded manifolds $\left(J^{r} Y, \mathscr{A}_{r}\right)$. It contains the subalgebra $\mathscr{O}_{\infty}^{*} Y$ of all exterior forms on jet manifolds $J^{r} Y$. It is an $\mathscr{O}_{\infty}^{0} Y$-algebra locally generated by elements $\left(c_{\Lambda}^{a}, d x^{\lambda}, d y_{\Lambda}^{i}, d c_{\Lambda}^{a}\right), 0 \leq|\Lambda|$. The collective symbol $\left(s^{A}\right)$ further stands for the tuple $\left(y^{i}, c^{a}\right)$, called a local basis for the DGA $\mathscr{S}_{\infty}^{*}[F ; Y]$. Let $[A]=\left[s^{A}\right]$ denote the Grassmann parity.

With this notation, a graded derivation of the $\mathbb{R}$-ring $\mathscr{S}_{\infty}^{0}[F ; Y]$ takes the form

$$
\left.\vartheta=\vartheta^{\lambda} \partial_{\lambda}+\sum_{0 \leq|\Lambda|} \vartheta_{\Lambda}^{A} \partial_{A}^{\Lambda}, \quad \partial_{A}^{\Lambda}\left(s_{\Sigma}^{B}\right)=\partial_{A}^{\Lambda}\right\rfloor d s_{\Sigma}^{B}=\delta_{A}^{B} \delta_{\Sigma}^{\Lambda}
$$

It yields the Lie derivative $\left.\left.\mathbf{L}_{\vartheta} \phi=\vartheta\right\rfloor d \phi+d(\vartheta\rfloor \phi\right)$ of the DGA $\mathscr{S}_{\infty}^{*}[F ; Y]$. In particular, the total derivatives are defined as the derivations

$$
d_{\lambda}=\partial_{\lambda}+\sum_{0 \leq|\Lambda|} s_{\lambda+\Lambda}^{A} \partial_{A}^{\Lambda}, \quad d_{\Lambda}=d_{\lambda_{1}} \cdots d_{\lambda_{k}}, \quad \lambda+\Lambda=\left(\lambda \lambda_{1} \ldots \lambda_{k}\right) .
$$


The DGA $\mathscr{S}_{\infty}^{*}[F ; Y]$ is split into the Grassmann-graded variational bicomplex of modules $\mathscr{S}_{\infty}^{k, r}[F ; Y]$ of $r$-horizontal and $k$-contact graded forms locally generated by one-forms $d x^{\lambda}$ and $\theta_{\Lambda}^{A}=d s_{\Lambda}^{A}-s_{\lambda+\Lambda}^{A} d x^{\lambda}$ [1, 3, 4, 8]. It contains the variational subcomplex

$$
0 \rightarrow \mathbb{R} \longrightarrow \mathscr{S}_{\infty}^{0}[F ; Y] \stackrel{d_{H}}{\longrightarrow} \mathscr{S}_{\infty}^{0,1}[F ; Y] \cdots \stackrel{d_{H}}{\longrightarrow} \mathscr{S}_{\infty}^{0, n}[F ; Y] \stackrel{\delta}{\longrightarrow} \mathscr{S}_{\infty}^{1, n}[F ; Y],
$$

where $d_{H}(\phi)=d x^{\lambda} \wedge \mathbf{L}_{d_{\lambda}}(\phi)$ is the total differential and $\delta$ is the variational operator. Lagrangians and Euler-Lagrange operators are defined as its even elements

$$
\begin{aligned}
& L=\mathscr{L} d^{n} x \in \mathscr{S}_{\infty}^{0, n}[F ; Y], \\
& \delta L=\theta^{A} \wedge \mathscr{E}_{A} d^{n} x=\sum_{0 \leq|\Lambda|}(-1)^{|\Lambda|} \theta^{A} \wedge d_{\Lambda}\left(\partial_{A}^{\Lambda} \mathscr{L}\right) d^{n} x,
\end{aligned}
$$

The relevant cohomology of the variational bicomplex has been obtained [8, 11]. In particular, any variationally trivial ( $\delta$-closed) odd density $L \in \mathscr{S}_{\infty}^{0, n}[F ; Y]$ is $d_{H^{-}}$ exact, and the form $d L-\delta L$ is $d_{H}$-exact for any density $L \in \mathscr{S}_{\infty}^{0, n}[F ; Y]$.

A graded derivation $\vartheta$ (1) is called a variational symmetry of a Lagrangian $L$ if the Lie derivative $\mathbf{L}_{\vartheta} L$ is $d_{H}$-exact. We restrict our consideration to vertical contact graded derivations $\vartheta$ vanishing on $C^{\infty}(X) \subset \mathscr{S}_{\infty}^{0}[F ; Y]$ and preserving the ideal of contact forms of the DGA $\mathscr{S}_{\infty}^{*}[F ; Y]$. Such a derivation is the jet prolongation

$$
\vartheta=v^{A} \partial_{A}+\sum_{0<|\Lambda|} d_{\Lambda} v^{A} \partial_{A}^{\Lambda}
$$

of its restriction $v=v^{A} \partial_{A}$ to the ring $\mathscr{S}_{\infty}^{0}[F ; Y]$. It obeys the relations $\left.\vartheta\right\rfloor d_{H} \phi=$ $\left.-d_{H}(\vartheta\rfloor \phi\right), \phi \in \mathscr{S}_{\infty}^{*}[F ; Y]$. In particular, we have

$$
\left.\mathbf{L}_{\vartheta} L=v\right\rfloor \delta L+d_{H} \sigma=v^{A} \mathscr{E}_{A} d^{n} x+d_{H} \sigma
$$

for any Lagrangian $L$. It follows that $\vartheta$ (4) is a variational symmetry of $L$ iff the form $v\rfloor \delta L$ is $d_{H}$-exact. A graded derivation $\vartheta(4)$ is called nilpotent if $\mathbf{L}_{\vartheta}\left(\mathbf{L}_{\vartheta} \phi\right)=0$ for any horizontal form $\phi \in \mathscr{S}_{\infty}^{0, *}[F ; Y]$. It is nilpotent only if it is odd and iff $\vartheta(v)=0$.

For the sake of simplicity, the common symbol $v$ further stands for the graded derivation $\vartheta$ (4), its summand $v$, and the Lie derivative $\mathbf{L}_{\vartheta}$. We agree to call $v$ the graded derivation of the DGA $\mathscr{S}_{\infty}^{*}[F ; Y]$. Its right graded derivations $\overleftarrow{v}=\overleftarrow{\partial}_{A} v^{A}$ are also considered. One associates to any right graded derivation $\overleftarrow{v}=\overleftarrow{\partial}_{A} v^{A}$ the left one

$$
v^{l}=(-1)^{[v][A]} v^{A} \partial_{A}, \quad v^{l}(f)=(-1)^{[v][f]} v(f), \quad f \in \mathscr{S}_{\infty}^{0}[F ; Y] .
$$

\section{KT complex of Noether identities}

Let us start with the following notation. Given a vector bundle $E \rightarrow X$, we call $\bar{E}=E^{*} \otimes \wedge^{n} T^{*} X$ the density-dual of $E$. The density dual of a graded vector bundle 
$E=E^{0} \oplus E^{1}$ is $\bar{E}=\bar{E}^{1} \oplus \bar{E}^{0}$. Given a graded vector bundle $E=E^{0} \oplus E^{1}$ over $Y$, we consider the composite bundle $E \rightarrow E^{0} \rightarrow X$ and denote $\mathscr{P}_{\infty}^{*}[E ; Y]=\mathscr{S}_{\infty}^{*}\left[E ; E^{0}\right]$.

Given a Lagrangian theory $\left(\mathscr{S}_{\infty}^{*}[F ; Y], L\right)$, its Euler-Lagrange operator $\delta L(3)$ obeys NI defined by linear differential operators on the $\mathscr{S}_{\infty}^{0}[F ; Y]$-module $\mathscr{S}_{\infty}^{1, n}[F ; Y]$ whose kernels contain $\delta L$. To describe these NI let us consider the density-dual $\overline{V F}=V^{*} F \otimes_{F} \wedge{ }^{n} T^{*} X$ of the vertical tangent bundle $V F \rightarrow F$. We assume that $F \rightarrow Y$ is a trivial vector bundle. In this case,

$$
\overline{V F}=\left(\bar{F} \oplus V^{*} Y \underset{Y}{\otimes} \wedge T^{*} X\right) \oplus F
$$

is a graded vector bundle over $Y$. Let us enlarge $\mathscr{S}_{\infty}^{*}[F ; Y]$ to the DGA $\mathscr{P}_{\infty}^{*}[\overline{V F} ; Y]$ with a local basis $\left(s^{A}, \bar{s}_{A}\right),\left[\bar{s}_{A}\right]=([A]+1) \bmod 2$. Its elements $\bar{s}_{A}$ are called antifields of antifield number Ant $\left[\bar{s}_{A}\right]=1$. The DGA $\mathscr{P}_{\infty}^{*}[\overline{V F} ; Y]$ is endowed with the nilpotent right graded derivation $\bar{\delta}=\overleftarrow{\partial}^{A} \mathscr{E}_{A}$. Then we have the chain complex

$$
0 \leftarrow \operatorname{Im} \bar{\delta} \stackrel{\bar{\delta}}{\longleftarrow} \mathscr{P}_{\infty}^{0, n}[\overline{V F} ; Y]_{1} \stackrel{\bar{\delta}}{\longleftarrow} \mathscr{P}_{\infty}^{0, n}[\overline{V F} ; Y]_{2}
$$

of graded densities of antifield number $\leq 2$. Its one-chains are linear differential operators on $\mathscr{S}_{\infty}^{1, n}[F ; Y]$, and its one-cycles define the NI

$$
\begin{aligned}
& \bar{\delta} \Phi=0, \quad \Phi=\sum_{0 \leq|\Lambda|} \Phi^{A, \Lambda} \bar{s}_{\Lambda A} d^{n} x \in \mathscr{P}_{\infty}^{0, n}[\overline{V F} ; Y]_{1}, \\
& \sum_{0 \leq|\Lambda|} \Phi^{A, \Lambda} d_{\Lambda} \mathscr{E}_{A} d^{n} x=0 .
\end{aligned}
$$

Conversely, all NI (8) come from the cycles (7). In particular, one-chains $\Phi \in$ $\mathscr{P}_{\infty}^{0, n}[\overline{V F} ; Y]_{1}$ are necessarily NI if they are boundaries. Therefore, these NI are called trivial. Accordingly, non-trivial NI modulo the trivial ones correspond to elements of the first homology $H_{1}(\bar{\delta})$ of the complex (6) [4]. A Lagrangian $L$ is called degenerate if there are non-trivial NI.

Non-trivial NI obey first-stage NI. To describe them, let us assume that the module $H_{1}(\bar{\delta})$ is finitely generated. Namely, there exists a projective $C^{\infty}(X)$-module $\mathscr{C}_{(0)} \subset H_{1}(\bar{\delta})$ of finite rank possessing a local basis $\left\{\Delta_{r}\right\}$ such that any element $\Phi \in H_{1}(\bar{\delta})$ factorizes

$$
\Phi=\sum_{0 \leq|\Xi|} G^{r, \Xi} d_{\Xi} \Delta_{r} d^{n} x, \quad \Delta_{r}=\sum_{0 \leq|\Lambda|} \Delta_{r}^{A, \Lambda} \bar{s}_{\Lambda A}, \quad G^{r, \Xi}, \Delta_{r}^{A, \Lambda} \in \mathscr{S}_{\infty}^{0}[F ; Y],
$$

via elements of $\mathscr{C}_{(0)}$. Thus, all non-trivial NI (8) result from the NI

$$
\bar{\delta} \Delta_{r}=\sum_{0 \leq|\Lambda|} \Delta_{r}^{A, \Lambda} d_{\Lambda} \mathscr{E}_{A}=0
$$


By virtue of the Serre-Swan theorem, the module $\mathscr{C}_{(0)}$ is isomorphic to a module of sections of the density-dual $\bar{E}_{0}$ of some graded vector bundle $E_{0} \rightarrow X$. Let us enlarge $\mathscr{P}_{\infty}^{*}[\overline{V F} ; Y]$ to the DGA $\overline{\mathscr{P}}_{\infty}^{*}\{0\}=\mathscr{P}_{\infty}^{*}\left[\overline{V F} \oplus_{Y} \bar{E}_{0} ; Y\right]$ possessing a local basis $\left(s^{A}, \bar{s}_{A}, \bar{c}_{r}\right)$ of Grassmann parity $\left[\bar{c}_{r}\right]=\left(\left[\Delta_{r}\right]+1\right) \bmod 2$ and antifield number Ant $\left[\bar{c}_{r}\right]=$

2. This DGA is provided with the odd right graded derivation $\delta_{0}=\bar{\delta}+\overleftarrow{\partial}^{r} \Delta_{r}$ which is nilpotent iff the NI (10) hold. Then we have the chain complex

$$
0 \leftarrow \operatorname{Im} \bar{\delta} \stackrel{\bar{\delta}}{\leftarrow} \mathscr{P}_{\infty}^{0, n}[\overline{V F} ; Y]_{1} \stackrel{\delta_{0}}{\leftarrow} \overline{\mathscr{P}}_{\infty}^{0, n}\{0\}_{2} \stackrel{\delta_{0}}{\leftarrow} \overline{\mathscr{P}}_{\infty}^{0, n}\{0\}_{3}
$$

of graded densities of antifield number $\leq 3$. It possesses trivial homology $H_{0}\left(\delta_{0}\right)$ and $H_{1}\left(\delta_{0}\right)$. Its two-cycles define the first-stage NI

$$
\begin{aligned}
& \delta_{0} \Phi=0, \quad \Phi=G+H=\sum_{0 \leq|\Lambda|} G^{r, \Lambda} \bar{c}_{\Lambda r} d^{n} x+\sum_{0 \leq|\Lambda|,|\Sigma|} H^{(A, \Lambda)(B, \Sigma)} \bar{s}_{\Lambda A} \bar{s}_{\Sigma B} d^{n} x \\
& \sum_{0 \leq|\Lambda|} G^{r, \Lambda} d_{\Lambda} \Delta_{r} d^{n} x=-\bar{\delta} H
\end{aligned}
$$

However, the converse need not be true. One can show that NI (12) are cycles iff any $\bar{\delta}$-cycle $\Phi \in \mathscr{P}_{\infty}^{0, n}[\overline{V F} ; Y]_{2}$ is a $\delta_{0}$-boundary [4]. In particular, a cycle $\Phi$ is a boundary if its summand $G$ is $\bar{\delta}$-exact. Any boundary $\Phi \in \overline{\mathscr{P}}_{\infty}^{0, n}\{0\}_{2}$ necessarily defines firststage NI (12), called trivial. Accordingly, non-trivial first-stage NI modulo the trivial ones are identified to elements of the second homology $\mathrm{H}_{2}\left(\delta_{0}\right)$ of the complex (111). Note that this definition is independent on specification of a generating module $\mathscr{C}_{(0)}$. Given a different one, there exists a chain isomorphism between the corresponding complexes (11).

A degenerate Lagrangian is called reducible if there are non-trivial first-stage NI. These obey second-stage NI, and so on. Iterating the arguments, we say that a degenerate Lagrangian is $N$-stage reducible if the following hold [4].

(i) There are graded vector bundles $E_{0}, \ldots, E_{N}$ over $X$, and the DGA $\mathscr{P}_{\infty}^{*}[\overline{V F} ; Y]$ is enlarged to the DGA

$$
\overline{\mathscr{P}}_{\infty}^{*}\{N\}=\mathscr{P}_{\infty}^{*}\left[\overline{V F} \oplus_{Y} \bar{E}_{0} \underset{Y}{\oplus} \cdots \underset{Y}{\oplus} \bar{E}_{N} ; Y\right]
$$

with a local basis $\left(s^{A}, \bar{s}_{A}, \bar{c}_{r}, \bar{c}_{r_{1}}, \ldots, \bar{c}_{r_{N}}\right)$ of antifield number Ant $\left[\bar{c}_{r_{k}}\right]=k+2$.

(ii) The DGA (13) is provided with the nilpotent right graded derivation

$$
\begin{aligned}
\delta_{K T} & =\overleftarrow{\partial} A \mathscr{E}_{A}+\sum_{0 \leq|\Lambda|} \overleftarrow{\partial}^{r} \Delta_{r}^{A, \Lambda} \bar{s}_{\Lambda A}+\sum_{1 \leq k \leq N} \overleftarrow{\partial}^{r_{k}} \Delta_{r_{k}}, \\
\Delta_{r_{k}} & =\sum_{0 \leq|\Lambda|} \Delta_{r_{k}}^{r_{k-1}, \Lambda} \bar{c}_{\Lambda r_{k-1}}+\sum_{0 \leq|\Sigma|,|\Xi|}\left(h_{r_{k}}^{\left(r_{k-2}, \Sigma\right)(A, \Xi)} \bar{c}_{\Sigma r_{k-2}} \bar{s}_{\Xi A}+\ldots\right)
\end{aligned}
$$

of antifield number -1 , where the index $k=-1$ stands for $\bar{s}_{A}$. 
(iii) The module $\mathscr{\mathscr { P }}_{\infty}^{0, n}\{N\}_{\leq N+3}$ of densities of antifield number $\leq(N+3)$ is split into the exact $\mathrm{KT}$ chain complex

$$
\begin{aligned}
0 \leftarrow & \operatorname{Im} \bar{\delta} \stackrel{\bar{\delta}}{\longleftarrow} \mathscr{P}_{\infty}^{0, n}[\overline{V F} ; Y]_{1} \stackrel{\delta_{0}}{\longleftarrow} \overline{\mathscr{P}}_{\infty}^{0, n}\{0\}_{2} \stackrel{\delta_{1}}{\longleftarrow} \overline{\mathscr{P}}_{\infty}^{0, n}\{1\}_{3} \ldots \\
& \stackrel{\delta_{N-1}}{\longleftarrow} \overline{\mathscr{P}}_{\infty}^{0, n}\{N-1\}_{N+1} \stackrel{\delta_{K T}}{\longleftarrow} \overline{\mathscr{P}}_{\infty}^{0, n}\{N\}_{N+2} \stackrel{\delta_{K T}}{\longleftarrow} \overline{\mathscr{P}}_{\infty}^{0, n}\{N\}_{N+3}
\end{aligned}
$$

which satisfies the following homology condition.

DEFINITION 1. One says that the homology regularity condition holds if any $\delta_{k<N^{-}}$ cycle $\phi \in \overline{\mathscr{P}}_{\infty}^{0, n}\{k\}_{k+3} \subset \overline{\mathscr{P}}_{\infty}^{0, n}\{k+1\}_{k+3}$ is a $\delta_{k+1}$-boundary.

THEOREM 2. Given an N-reducible Lagrangian, the nilpotentness $\delta_{K T}^{2}=0$ of the KT operator (14) is equivalent to the non-trivial NI (10) and $(k \leq N)$-stage NI

$$
\sum_{0 \leq|\Lambda|} \Delta_{r_{k}}^{r_{k-1}, \Lambda} d_{\Lambda}\left(\sum_{0 \leq|\Sigma|} \Delta_{r_{k-1}}^{r_{k-2}, \Sigma} \bar{c}_{\Sigma r_{k-2}}\right)=-\overline{\boldsymbol{\delta}}\left(\sum_{0 \leq|\Sigma|,|\Xi|} h_{r_{k}}^{\left(r_{k-2}, \Sigma\right)(A, \Xi)} \bar{c}_{\Sigma r_{k-2}} \bar{S}_{\Xi A}\right) .
$$

\section{Inverse second Noether theorem}

A gauge symmetry of a Lagrangian $L$ is defined as a linear differential operator on some projective $C^{\infty}(X)$-module of finite rank with values in the module of variational symmetries of $L$. It can be described as follows [2, 3]. Let $E_{0} \rightarrow X$ be a graded vector bundle. Let us enlarge the DGA $\mathscr{S}_{\infty}^{*}[F ; Y]$ to the DGA $\mathscr{P}_{\infty}^{*}\left[F \oplus_{Y} E_{0} ; Y\right]$ possessing a local basis $\left(s^{A}, c^{r}\right)$ whose elements are called ghosts. A gauge symmetry of $L$ is an odd vertical contact graded derivation $u$ of $\mathscr{P}_{\infty}^{0}\left[F \oplus_{Y} E_{0} ; Y\right]$ which is a variational symmetry of $L$. Any Lagrangian admits gauge symmetries. Let $u_{(1)}$ be a differential operator on some projective $C^{\infty}(X)$-module of finite rank such that $u \circ u_{(1)}$ is $\bar{\delta}$-exact. It is called the first-stage gauge symmetry, and so on.

Different variants of the second Noether theorem relate reducible NI and gauge symmetries [1, 2, 3, 7]. Given the DGA $\overline{\mathscr{P}}_{\infty}^{*}\{N\}$ (13), let us consider the DGA

$$
\mathscr{P}_{\infty}^{*}\{N\}=\mathscr{P}_{\infty}^{*}\left[F \underset{Y}{\oplus_{Y}} E_{0} \underset{Y}{\bigoplus_{Y}} \cdots \underset{Y}{\bigoplus_{N}} E_{N} ; Y\right]
$$

possessing a local basis $\left(s^{A}, c^{r}, c^{r_{1}}, \ldots, c^{r_{N}}\right),\left[c^{r_{k}}\right]=\left(\left[\bar{c}_{r_{k}}\right]+1\right) \bmod 2$, and the DGA

$$
P_{\infty}^{*}\{N\}=\mathscr{P}_{\infty}^{*}\left[\overline{V F} \underset{Y}{\oplus} E_{0} \oplus \cdots \underset{Y}{\oplus} E_{N} \underset{Y}{\oplus} \bar{E}_{0} \underset{Y}{\oplus} \cdots \underset{Y}{\oplus} \bar{E}_{N} ; Y\right]
$$

with a local basis $\left(s^{A}, \bar{s}^{A}, c^{r}, c^{r_{1}}, \ldots, c^{r_{N}}, \bar{c}_{r}, \bar{c}_{r_{1}}, \ldots, \bar{c}_{r_{N}}\right)$. Their elements $c^{r_{k}}$ are called ghosts of ghost number $\operatorname{gh}\left[c^{r_{k}}\right]=k+1$ and antifield number Ant $\left[c^{r_{k}}\right]=-(k+1)$. 
The DGAs $\overline{\mathscr{P}}_{\infty}^{*}\{N\}(13)$ and $\mathscr{P}_{\infty}^{*}\{N\}$ (18) are subalgebras of $P_{\infty}^{*}\{N\}$ (19). The KT operator $\delta_{K T}$ (14) is naturally extended to a graded derivation of the DGA $P_{\infty}^{*}\{N\}$.

THEOREM 3. Given the KT complex (16), the module of graded densities $\mathscr{P}_{\infty}^{0, n}\{N\}$ is split into the cochain sequence

$$
\begin{aligned}
0 & \rightarrow \mathscr{S}_{\infty}^{0, n}[F ; Y] \stackrel{u_{e}}{\longrightarrow} \mathscr{P}_{\infty}^{0, n}\{N\}^{1} \stackrel{u_{e}}{\longrightarrow} \mathscr{P}_{\infty}^{0, n}\{N\}^{2} \stackrel{u_{e}}{\longrightarrow} \cdots, \\
u_{e} & =u+u_{(1)}+\cdots+u_{(N)}=u^{A} \frac{\partial}{\partial s^{A}}+u^{r} \frac{\partial}{\partial c^{r}}+\cdots+u^{r_{N-1}} \frac{\partial}{\partial c^{r_{N-1}}},
\end{aligned}
$$

graded in the ghost number, where $u(29)$ and $u_{(k)}(31), k=1, \ldots, N$, are the gauge and higher-stage gauge symmetries of an original Lagrangian $L$.

Proof. Note that any tuple $\left(f^{\prime}, f^{\Lambda}\right),|\Lambda| \leq k$, of local graded functions $f^{\prime}, f^{\Lambda} \in$ $\mathscr{S}_{\infty}^{0}[F ; Y]$ obeys the relations

$$
\begin{aligned}
& \sum_{0 \leq|\Lambda| \leq k} f^{\Lambda} d_{\Lambda} f^{\prime} d^{n} x=\sum_{0 \leq|\Lambda| \leq k}(-1)^{|\Lambda|} d_{\Lambda}\left(f^{\Lambda}\right) f d^{n} x+d_{H} \sigma, \\
& \sum_{0 \leq|\Lambda| \leq k}(-1)^{|\Lambda|} d_{\Lambda}\left(f^{\Lambda} \phi\right)=\sum_{0 \leq|\Lambda| \leq k} \eta(f)^{\Lambda} d_{\Lambda} \phi, \\
& \eta(f)^{\Lambda}=\sum_{0 \leq|\Sigma| \leq k-|\Lambda|}(-1)^{|\Sigma+\Lambda|} \frac{(|\Sigma+\Lambda|) !}{|\Sigma| !|\Lambda| !} d_{\Sigma} f^{\Sigma+\Lambda}, \quad(\eta \circ \eta)(f)^{\Lambda}=f^{\Lambda} .
\end{aligned}
$$

Let us extend an original Lagrangian $L$ to the Lagrangian

$$
L_{e}=L+L_{1}=L+\sum_{0 \leq k \leq N} c^{r_{k}} \Delta_{r_{k}} d^{n} x=L+\delta_{K T}\left(\sum_{0 \leq k \leq N} c^{r_{k}} \bar{c}_{r_{k}} d^{n} x\right)
$$

of zero antifield number. It is readily observed that the KT operator $\delta_{K T}$ is a variational symmetry of $L_{e}$. It follows that

$$
\begin{aligned}
& {\left[\frac{\overleftarrow{\delta} \mathscr{L}_{e}}{\delta \bar{s}_{A}} \mathscr{E}_{A}+\sum_{0 \leq k \leq N} \frac{\overleftarrow{\delta} \mathscr{L}_{e}}{\delta \bar{c}_{r_{k}}} \Delta_{r_{k}}\right] d^{n} x=\left[v^{A} \mathscr{E}_{A}+\sum_{0 \leq k \leq N} v^{r_{k}} \frac{\delta \mathscr{L}_{e}}{\delta c^{r_{k}}}\right] d^{n} x=d_{H} \sigma} \\
& v^{A}=\frac{\overleftarrow{\delta} \mathscr{L}_{e}}{\delta \bar{s}_{A}}=u^{A}+w^{A}=\sum_{0 \leq|\Lambda|} c_{\Lambda}^{r} \eta\left(\Delta_{r}^{A}\right)^{\Lambda}+\sum_{1 \leq i \leq N} \sum_{0 \leq|\Lambda|} c_{\Lambda}^{r_{i}} \eta\left(\overleftarrow{\partial^{A}}\left(h_{r_{i}}\right)\right)^{\Lambda} \\
& v^{r_{k}}=\frac{\overleftarrow{\delta} \mathscr{L}_{e}}{\delta \bar{c}_{r_{k}}}=u^{r_{k}}+w^{r_{k}}=\sum_{0 \leq|\Lambda|} c_{\Lambda}^{r_{k+1}} \eta\left(\Delta_{r_{k+1}}^{r_{k}}\right)^{\Lambda}+\sum_{k+1<i \leq N} \sum_{0 \leq|\Lambda|} c_{\Lambda}^{r_{i}} \eta\left(\overleftarrow{\partial}^{r_{k}}\left(h_{r_{i}}\right)\right)^{\Lambda}
\end{aligned}
$$

The equality (26) falls into the set of equalities

$$
\begin{aligned}
& \stackrel{\overleftarrow{\delta}\left(c^{r} \Delta_{r}\right)}{\delta \bar{s}_{A}} \mathscr{E}_{A} d^{n} x=u^{A} \mathscr{E}_{A} d^{n} x=d_{H} \sigma_{0} \\
& {\left[\frac{\overleftarrow{\delta}\left(c^{r_{k}} \Delta_{r_{k}}\right)}{\delta \bar{s}_{A}} \mathscr{E}_{A}+\sum_{0 \leq i<k} \frac{\overleftarrow{\delta}\left(c^{r_{k}} \Delta_{r_{k}}\right)}{\delta \bar{c}_{r_{i}}} \Delta_{r_{i}}\right] d^{n} x=d_{H} \sigma_{k}, \quad k=1, \ldots, N}
\end{aligned}
$$


By virtue of the equality (27) and the formula (5), the graded derivation

$$
u=u^{A} \frac{\partial}{\partial s^{A}}, \quad u^{A}=\sum_{0 \leq|\Lambda|} c_{\Lambda}^{r} \eta\left(\Delta_{r}^{A}\right)^{\Lambda},
$$

of $\mathscr{P}^{0}\{0\}$ is a variational and, consequently, gauge symmetry of a Lagrangian $L$ [3, 8]. Every equality (28) falls into a set of equalities graded by the polynomial degree in antifields. Let us consider the equality, linear in antifields $\bar{c}_{r_{k-2}}$. We have

$$
\begin{aligned}
& {\left[\frac{\overleftarrow{\delta}}{\delta \bar{s}_{A}}\left(c^{r_{k}} \sum_{0 \leq|\Sigma|,|\Xi|} h_{r_{k}}^{\left(r_{k-2}, \Sigma\right)(A, \Xi)} \bar{c}_{\Sigma r_{k-2}} \bar{s}_{\Xi A}\right) \mathscr{E}_{A}+\right.} \\
& \left.\quad \stackrel{\overleftarrow{\delta}}{\quad}\left(c^{r_{k}} \sum_{0 \leq|\Sigma|} \Delta_{r_{k}}^{r_{k-1}^{\prime}, \Sigma} \bar{c}_{\Sigma r_{k-1}^{\prime}}\right) \sum_{0 \leq|\Xi|} \Delta_{r_{k-1}}^{r_{k-2}, \Xi} \bar{c}_{\Xi r_{k-2}}\right] d^{n} x=d_{H} \sigma_{k}
\end{aligned}
$$

This equality is brought into the form

$$
\left[\sum_{0 \leq|\Xi|}(-1)^{|\Xi|} d_{\Xi}\left(c^{r_{k}} \sum_{0 \leq|\Sigma|} h_{r_{k}}^{\left(r_{k-2}, \Sigma\right)(A, \Xi)} \bar{c}_{\Sigma r_{k-2}}\right) \mathscr{E}_{A}+u^{r_{k-1}} \sum_{0 \leq|\Xi|} \Delta_{r_{k-1}}^{r_{k-2}, \Xi} \bar{c}_{\Xi r_{k-2}}\right] d^{n} x=d_{H} \sigma_{k} .
$$

Using the relation (22), we obtain the equality

$$
\left[\sum_{0 \leq|\Xi|} c^{r_{k}} \sum_{0 \leq|\Sigma|} h_{r_{k}}^{\left(r_{k-2}, \Sigma\right)(A, \Xi)} \bar{c}_{\Sigma r_{k-2}} d_{\Xi} \mathscr{E}_{A}+u^{r_{k-1}} \sum_{0 \leq|\Xi|} \Delta_{r_{k-1}}^{r_{k-2}, \Xi} \bar{c}_{\Xi r_{k i-2}}\right] d^{n} x=d_{H} \sigma_{k}^{\prime} .
$$

The variational derivative of both its sides with respect to $\bar{c}_{r_{k-2}}$ leads to the relation

$$
\begin{aligned}
& \sum_{0 \leq|\Sigma|} d_{\Sigma} u^{r_{k-1}} \frac{\partial}{\partial c_{\Sigma}^{r_{k-1}}} u^{r_{k-2}}=\bar{\delta}\left(\alpha^{r_{k-2}}\right), \\
& \alpha^{r_{k-2}}=-\sum_{0 \leq|\Sigma|} \eta\left(h_{r_{k}}^{\left(r_{k-2}\right)(A, \Xi)}\right)^{\Sigma} d_{\Sigma}\left(c^{r_{k}} \bar{S}_{\Xi A}\right) .
\end{aligned}
$$

This is the $k$-stage gauge symmetry condition [3]. Thus, the odd graded derivations

$$
u_{(k)}=u^{r_{k-1}} \frac{\partial}{\partial c^{r_{k-1}}}, \quad u^{r_{k-1}}=\sum_{0 \leq|\Lambda|} c_{\Lambda}^{r_{k}} \eta\left(\Delta_{r_{k}}^{r_{k-1}}\right)^{\Lambda}, \quad k=1, \ldots, N,
$$

are $k$-stage gauge symmetries. Graded derivations $u$ (29), $u_{(k)}$ (31) form the ascent gauge operator (20) of ghost number 1 . It provides the cochain sequence (20).

Following the proof of Theorem 3 , one can show that any $C^{\infty}(X)$-module of NI yields a gauge symmetry of a Lagrangian. Since the gauge operator 21) need not be nilpotent, the direct second Noether theorem can not be formulated in homology terms. Therefore, gauge and higher-stage gauge symmetries are said to be non-trivial if they are associated to non-trivial NI and higher-stage NI, respectively. 
With the gauge operator (21), the extended Lagrangian $L_{e}(25)$ takes the form

$$
L_{e}=L+u_{e}\left(\sum_{0 \leq k \leq N} c^{r_{k-1}} \bar{c}_{r_{k-1}}\right) d^{n} x+L_{1}^{*}+d_{H} \sigma,
$$

where $L_{1}^{*}$ is a term of polynomial degree in antifields exceeding 1 .

\section{KT-BRST complex}

The DGA $P_{\infty}^{*}\{N\}$ (19) exemplifies a field-antifield theory of the following type [1, 9]. Let $Z^{\prime} \rightarrow Z \rightarrow X$ be a composite bundle where $Z^{\prime} \rightarrow Z$ is a trivial vector bundle. Let us consider the DGA $\mathscr{P}_{\infty}^{*}\left[\overline{V Z^{\prime}} ; Z\right]$ with a local basis $\left(z^{a}, \bar{z}_{a}\right)$, where $\left[\bar{z}_{a}\right]=\left(\left[z^{a}\right]+1\right) \bmod 2$. Its elements $z^{a}$ and $\bar{z}_{a}$ are called fields and antifields, respectively. Densities of this DGA are endowed with the antibracket

$$
\left\{\mathbf{L} d^{n} x, \mathbf{L}^{\prime} d^{n} x\right\}=\left[\frac{\overleftarrow{\delta} \mathbf{L}}{\delta \bar{z}_{a}} \frac{\delta \mathbf{L}^{\prime}}{\delta z^{a}}+(-1)^{\left[\mathbf{L}^{\prime}\right]\left(\left[\mathbf{L}^{\prime}\right]+1\right)} \frac{\overleftarrow{\delta} \mathbf{L}^{\prime}}{\delta \bar{z}_{a}} \frac{\delta \mathbf{L}}{\delta z^{a}}\right] d^{n} x
$$

Furthermore, one associates to any Lagrangian $\mathbf{L} d^{n} x$ the odd graded derivations

$$
\begin{aligned}
& v_{\mathbf{L}}=\overleftarrow{\mathscr{E}}^{a} \partial_{a}=\frac{\overleftarrow{\delta} \mathbf{L}}{\delta \bar{z}_{a}} \frac{\partial}{\partial z^{a}}, \quad \bar{v}_{\mathbf{L}}=\overleftarrow{\partial}{ }^{a} \mathscr{E}_{a}=\frac{\overleftarrow{\partial}}{\partial \bar{z}_{a}} \frac{\delta \mathbf{L}}{\delta z^{a}} \\
& \vartheta_{\mathbf{L}}=v_{\mathbf{L}}+\bar{v}_{\mathbf{L}}^{l}=(-1)^{[a]+1}\left(\frac{\delta \mathbf{L}}{\delta \bar{z}^{a}} \frac{\partial}{\partial z_{a}}+\frac{\delta \mathbf{L}}{\delta z^{a}} \frac{\partial}{\partial \bar{z}_{a}}\right), \\
& \vartheta_{\mathbf{L}}\left(\mathbf{L}^{\prime} d^{n} x\right)=\left\{\mathbf{L} d^{n} x, \mathbf{L}^{\prime} d^{n} x\right\} .
\end{aligned}
$$

THEOREM 4. The following conditions are equivalent. (i) The antibracket of a Lagrangian $\mathbf{L} d^{n} x$ is $d_{H}$-exact, i.e.,

$$
\left\{\mathbf{L} d^{n} x, \mathbf{L} d^{n} x\right\}=2 \frac{\overleftarrow{\delta} \mathbf{L}}{\delta \bar{z}_{a}} \frac{\delta \mathbf{L}}{\delta z^{a}} d^{n} x=d_{H} \sigma
$$

(ii) The graded derivation $v$ (34) is a variational symmetry of a Lagrangian $\mathbf{L} d^{n} x$.

(iii) The graded derivation $\bar{v}$ (34) is a variational symmetry of $\mathbf{L} d^{n} x$. (iv) The graded derivation $\vartheta_{\mathbf{L}}$ (35) is nilpotent.

Proof. By virtue of the formula (5), conditions (ii) and (iii) are equivalent to condition (i). The equality (36) is equivalent to that the odd density $\overleftarrow{\mathscr{E}} \overleftarrow{\mathscr{E}}_{a} d^{n} x$ is variationally

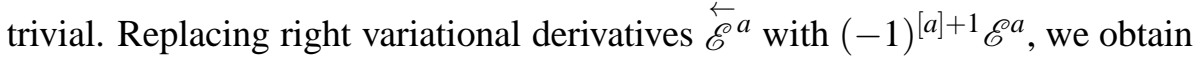

$$
2 \sum_{a}(-1)^{[a]} \mathscr{E}^{a} \mathscr{E}_{a} d^{n} x=d_{H} \sigma .
$$


The variational operator acting on this relation results in the equalities

$$
\begin{aligned}
& \left.\sum_{0 \leq|\Lambda|}(-1)^{[a]+|\Lambda|} d_{\Lambda}\left(\partial_{b}^{\Lambda}\left(\mathscr{E}^{a} \mathscr{E}_{a}\right)\right)=\sum_{0 \leq|\Lambda|}(-1)^{[a]}\left[\eta\left(\partial_{b} \mathscr{E}^{a}\right)^{\Lambda} \mathscr{E}_{\Lambda a}+\eta\left(\partial_{b} \mathscr{E}_{a}\right)^{\Lambda} \mathscr{E}_{\Lambda}\right)\right]=0, \\
& \sum_{0 \leq|\Lambda|}(-1)^{[a]+|\Lambda|} d_{\Lambda}\left(\partial^{\Lambda b}\left(\mathscr{E}^{a} \mathscr{E}_{a}\right)\right)=\sum_{0 \leq|\Lambda|}(-1)^{[a]}\left[\eta\left(\partial^{b} \mathscr{E}^{a}\right)^{\Lambda} \mathscr{E}_{\Lambda a}+\eta\left(\partial^{b} \mathscr{E}_{a}\right) \mathscr{E}_{\Lambda}^{a}\right]=0 .
\end{aligned}
$$

Due to the identity $(\delta \circ \delta)(L)=0, \eta\left(\partial_{B} \mathscr{E}_{A}\right)^{\Lambda}=(-1)^{[A][B]} \partial_{A}^{\Lambda} \mathscr{E}_{B}$, we obtain

$$
\begin{aligned}
& \sum_{0 \leq|\Lambda|}(-1)^{[a]}\left[(-1)^{[b]([a]+1)} \partial^{\Lambda a} \mathscr{E}_{b} \mathscr{E}_{\Lambda a}+(-1)^{[b][a]} \partial_{a}^{\Lambda} \mathscr{E}_{b} \mathscr{E}_{\Lambda} a\right]=0, \\
& \sum_{0 \leq|\Lambda|}(-1)^{[a]+1}\left[(-1)^{([b]+1)([a]+1)} \partial^{\Lambda a} \mathscr{E}^{b} \mathscr{E}_{\Lambda a}+(-1)^{([b]+1)[a]} \partial_{a}^{\Lambda} \mathscr{E}^{b} \mathscr{E}_{\Lambda}\right]=0,
\end{aligned}
$$

for all $\mathscr{E}_{b}$ and $\mathscr{E}^{b}$. This is exactly condition (iv).

The equality (36) is called the master equation. For instance, any variationally trivial Lagrangian satisfies the master equation.

Being an element of the DGA $P_{\infty}^{*}\{N\}$ (19), an original Lagrangian $L$ obeys the master equation (36) and yields the graded derivations $v_{L}=0, \bar{v}_{L}=\bar{\delta}$ (34). The graded derivations (34) associated to the Lagrangian $L_{e}(25)$ are extensions

$$
v_{e}=u_{e}+\frac{\overleftarrow{\delta} \mathscr{L}_{1}^{*}}{\delta \bar{s}_{A}} \frac{\partial}{\partial s^{A}}+\sum_{0 \leq k \leq N} \frac{\overleftarrow{\delta} \mathscr{L}_{1}^{*}}{\delta \bar{c}_{r_{k}}} \frac{\partial}{\partial c^{r_{k}}}, \quad \bar{v}_{e}=\delta_{K T}+\frac{\overleftarrow{\partial}}{\partial \bar{s}_{A}} \frac{\delta \mathscr{L}_{1}}{\delta s^{A}}
$$

of the gauge and KT operators, respectively. However, $L_{e}$ need not satisfy the master equation. Therefore, let us consider its extension

$$
L_{E}=L_{e}+L^{\prime}=L+L_{1}+L_{2}+\cdots
$$

by means of even densities $L_{i}, i \geq 2$, of zero antifield number and polynomial degree $i$ in ghosts. The corresponding graded derivations (34) read

$$
\begin{gathered}
v_{E}=v_{e}+\frac{\overleftarrow{\delta} \mathscr{L}^{\prime}}{\delta \bar{s}_{A}} \frac{\partial}{\partial s^{A}}+\sum_{0 \leq k \leq N} \frac{\overleftarrow{\delta} \mathscr{L}^{\prime}}{\delta \bar{c}_{r_{k}}} \frac{\partial}{\partial c^{r_{k}}} \\
\bar{v}_{E}=\bar{v}_{e}+\frac{\overleftarrow{\partial}}{\partial \bar{s}_{A}} \frac{\delta \mathscr{L}^{\prime}}{\delta s^{A}}+\sum_{0 \leq k \leq N} \frac{\overleftarrow{\partial}}{\partial \bar{c}_{r_{k}}} \frac{\delta \mathscr{L}^{\prime}}{\delta c^{r_{k}}}
\end{gathered}
$$

A Lagrangian $L_{E}$ (37) where $L+L_{1}=L_{e}$ is called a proper extension of an original Lagrangian $L$. The following is a corollary of Theorem 4

COROLLARY 5. A Lagrangian $L$ is extended to a proper solution $L_{E}$ (37) of the master equation only if the gauge operator $u_{e}(20)$ admits a nilpotent extension. 
By virtue of condition (iv) of Theorem 4 this nilpotent extension is the derivation $\vartheta_{E}=v_{E}+\bar{v}_{E}^{l}(35)$, called the KT-BRST operator. With this operator, the module of densities $P_{\infty}^{0, n}\{N\}$ is split into the KT-BRST complex

$$
\cdots \rightarrow P_{\infty}^{0, n}\{N\}_{2} \rightarrow P_{\infty}^{0, n}\{N\}_{1} \rightarrow P_{\infty}^{0, n}\{N\}_{0} \rightarrow P_{\infty}^{0, n}\{N\}^{1} \rightarrow P_{\infty}^{0, n}\{N\}^{2} \rightarrow \cdots .
$$

Putting all ghosts zero, we obtain a cochain morphism of this complex onto the KT complex, extended to $\mathscr{P}_{\infty}^{0, n}\{N\}$ and reversed into the cochain one. Letting all antifields zero, we come to a cochain morphism of the KT-BRST complex (40) onto the cochain sequence (20), where the gauge operator is extended to the antifield-free part of the KT-BRST operator.

THEOREM 6. If the gauge operator $u_{e}(20)$ can be extended to a nilpotent graded derivation

$$
u_{E}=u_{e}+\xi=u^{A} \partial_{A}+\sum_{1 \leq k \leq N}\left(u^{r_{k-1}}+\xi^{r_{k-1}}\right) \partial_{r_{k-1}}+\xi^{r_{N}} \partial_{r_{N}}
$$

of ghost number 1 by means of antifield-free terms of higher polynomial degree in ghosts, then the master equation has a proper solution

$$
L_{E}=L_{e}+\sum_{1 \leq k \leq N} \xi^{r_{k-1}} \bar{c}_{r_{k-1}} d^{n} x=L+u_{E}\left(\sum_{0 \leq k \leq N} c^{r_{k-1}} \bar{c}_{r_{k-1}}\right) d^{n} x+d_{H} \sigma .
$$

Proof. It is easily justified that, if the graded derivation $u_{E}$ (41) is nilpotent, then the right hand sides of the equalities (30) equal zero. Using the relations (22) - (24), one can show that, in this case, the right hand sides of the higher-stage NI (17) also equal zero [3]. It follows that the summand $G_{r_{k}}$ of each cocycle $\Delta_{r_{k}}$ (15) is $\delta_{k-1^{-}}$ closed. Then its summand $h_{r_{k}}$ is also $\delta_{k-1}$-closed and, consequently, $\delta_{k-2}$-closed. Hence it is $\delta_{k-1}$-exact by virtue of the homology regularity condition. Therefore, $\Delta_{r_{k}}$ contains only the term $G_{r_{k}}$ linear in antifields. It follows that the Lagrangian $L_{e}$ (25) and, consequently, the Lagrangian $L_{E}$ (42) are affine in antifields. In this case, we have $u^{A}=\overleftarrow{\delta}^{A}\left(\mathscr{L}_{e}\right), u^{r_{k}}=\overleftarrow{\delta}^{r_{k}}\left(\mathscr{L}_{e}\right)$ for all indices $A$ and $r_{k}$ and, consequently, $u_{E}^{A}=\overleftarrow{\delta}^{A}\left(\mathscr{L}_{E}\right), u_{E}^{r_{k}}=\overleftarrow{\delta}^{r_{k}}\left(\mathscr{L}_{E}\right)$, i.e., $u_{E}=v_{E}$ is the graded derivation (38) defined by the Lagrangian $L_{E}$. Its nilpotency condition takes the form $u_{E}\left(\overleftarrow{\delta}^{A}\left(\mathscr{L}_{E}\right)\right)=0$, $u_{E}\left(\overleftarrow{\delta}^{r_{k}}\left(\mathscr{L}_{E}\right)\right)=0$. Hence, we obtain

$$
u_{E}\left(\mathscr{L}_{E}\right)=u_{E}\left(\overleftarrow{\delta}^{A}\left(\mathscr{L}_{E}\right) \bar{s}_{A}+\overleftarrow{\delta}^{r_{k}}\left(\mathscr{L}_{E}\right) \bar{c}_{r_{k}}\right)=0
$$

i.e., $u_{E}$ is a variational symmetry of $L_{E}$. Consequently, $L_{E}$ obeys the master equation.

COROLLARY 7. The gauge operator (20) admits the nilpotent extension (41) only if gauge symmetry conditions (30) and the higher-stage NI (17) are satisfied off-shell. In this case, the Lagrangian $L_{e}(25)$ is affine in antifields. 
The nilpotent extension (41) of the gauge operator is called the BRST operator. It brings the cochain sequence (20) into the BRST complex.

By virtue of Corollary 7, the gauge operator $u_{e}$ is extended to the BRST operator only if gauge symmetry conditions hold off-shell, i.e., $u_{e}\left(u_{e}\right)=u\left(u_{e}\right)$. Let us consider a particular case when higher-stage gauge symmetries $u_{(k)}(31)$ are independent of original fields $s^{A}$, i.e., $u_{e}\left(u_{e}\right)=u(u)$. In this case, the BRST operator $u_{E}$ (41) reads

$$
u_{E}=u_{e}+\xi=u_{e}+\xi^{r} \partial_{r}, \quad(u+\xi)\left(u^{A}\right)=0, \quad(u+\xi)\left(\xi^{r}\right)=0, \quad u_{(1)}\left(\xi^{r}\right)=0 .
$$

For instance, irreducible theories and Abelian reducible theories, where $u(u)=0$, are of this type. One can think of the first and second conditions (43) as being the generalized commutation relations and the Jacobi identity, respectively [3]. It follows from the second one that $\xi$ is quadratic in ghosts. Moreover, if a gauge symmetry $u$ is polynomial in fields, then it is necessarily affine, and $\xi$ is independent of original fields. In Abelian reducible theories, the gauge operator $u_{e}$ itself is nilpotent. The corresponding proper solution (42) of the master equation reads

$$
L_{E}=L+\delta_{K T}\left(\sum_{0 \leq k \leq N} c^{r_{k}} \bar{c}_{r_{k}} d^{n} x\right)=L+u_{e}\left(\sum_{0 \leq k \leq N} c^{r_{k-1}} \bar{c}_{r_{k-1}}\right) d^{n} x+d_{H} \sigma .
$$

\section{Examples}

1) Yang-Mills gauge theory exemplifies an irreducible degenerate Lagrangian theory where $u_{E}$ (41) is the familiar BRST operator [9]. Let us consider Yang-Mills supergauge theory where gauge symmetries form a finite-dimensional Lie superalgebra over $C^{\infty}(X)$. Let $\mathscr{G}=\mathscr{G}^{0} \oplus \mathscr{G}^{1}$ be a real Lie superalgebra with a basis $\left\{e_{r}\right\}$, $r=1, \ldots, m$, and real structure constants $c_{i j}^{r}$. We denote $\left[e_{r}\right]=[r]$. Given the enveloping algebra $\overline{\mathscr{G}}$ of $\mathscr{G}$, let us assume that there is an invariant even element $h^{i j} e_{i} e_{j}$ of $\overline{\mathscr{G}}$ such that the matrix $h^{i j}$ is non-degenerate. The gauge theory of $\mathscr{G}$ on $X=\mathbb{R}^{n}$ is described by the DGA $\mathscr{S}^{*}[F ; Y]$ where

$$
F=(X \times \mathscr{G}) \otimes T^{*} X \rightarrow\left(X \times \mathscr{G}^{0}\right) \otimes T^{*} X \rightarrow X .
$$

Its basis is $\left(a_{\lambda}^{r}\right),\left[a_{\lambda}^{r}\right]=[r]$. The Yang-Mills Lagrangian reads

$$
L_{Y M}=\frac{1}{4} h_{i j} \eta^{\lambda \mu} \eta^{\beta v} \mathscr{F}_{\lambda \beta}^{i} \mathscr{F}_{\mu \nu}^{j} d^{n} x, \quad \mathscr{F}_{\lambda \mu}^{r}=a_{\lambda \mu}^{r}-a_{\mu \lambda}^{r}+c_{i j}^{r} a_{\lambda}^{i} a_{\mu}^{j} .
$$

Its Euler-Lagrange operator obeys the irreducible NI

$$
c_{j i}^{r} a_{\lambda}^{i} \mathscr{E}_{r}^{\lambda}+d_{\lambda} \mathscr{E}_{j}^{\lambda}=0 .
$$

Therefore, we enlarge $\mathscr{S}^{*}[F ; Y]$ to the DGA

$$
P^{*}\{0\}=\mathscr{P}^{*}\left[\overline{V F} \oplus_{Y} E_{0} \oplus_{Y} \bar{E}_{0} ; Y\right], \quad E_{0}=X \times \mathscr{G}_{0},
$$


whose basis $\left(a_{\lambda}^{r}, c^{r}, \bar{a}_{r}^{\lambda}, \bar{c}_{r}\right),\left[c^{r}\right]=([r]+1) \bmod 2,\left[\bar{a}_{r}^{\lambda}\right]=\left[\bar{c}_{r}\right]=[r]$, contains ghosts $c^{r}$ of ghost number 1 and antifields $\bar{a}_{r}^{\lambda}, \bar{c}_{r}$ of antifield numbers 1 and 2, respectively. Then the gauge operator $u_{e}(20)$ and the Lagrangian $L_{e}(25)$ read

$$
u_{e}=\left(-c_{j i}^{r} c^{j} a_{\lambda}^{i}+c_{\lambda}^{r}\right) \frac{\partial}{\partial a_{\lambda}^{r}}, \quad L_{e}=L_{Y M}+\left(-c_{j i}^{r} c^{j} a_{\lambda}^{i}+c_{\lambda}^{r}\right) \bar{a}_{r}^{\lambda} d^{n} x
$$

The gauge operator $u_{e}$ admits the BRST extension

$$
u_{E}=u_{e}+\xi=\left(-c_{j i}^{r} c^{j} a_{\lambda}^{i}+c_{\lambda}^{r}\right) \frac{\partial}{\partial a_{\lambda}^{r}}-\frac{1}{2}(-1)^{[i]} c_{i j}^{r} c^{i} c^{j} \frac{\partial}{\partial c^{r}} .
$$

Then the proper solution (42) of the master equation takes the form

$$
L_{E}=L_{Y M}+\left(-c_{i j}^{r} c^{j} a_{\lambda}^{i}+c_{\lambda}^{r}\right) \bar{a}_{r}^{\lambda} d^{n} x-\frac{1}{2}(-1)^{[i]} c_{i j}^{r} c^{i} c^{j} \bar{c}_{r} d^{n} x .
$$

2) In contrast with Yang-Mills gauge theory, gauge symmetries of gravitation theory fail to form a finite-dimensional Lie algebra. Gravitation theory can be formulated as gauge theory on natural bundles over a four-dimensional manifold $X$. These bundles admit the functorial lift $\widetilde{\tau}$ of any vector field $\tau$ on $X$ such that $\tau \mapsto \bar{\tau}$ is a Lie algebra monomorphism. This lift is an infinitesimal generator of a local one-parameter group of general covariant transformations. Dynamic variables of gauge gravitation theory are linear connections and pseudo-Riemannian metrics on $X$. The first ones are principal connections on the linear frame bundle $L X$ of $X$. They are represented by sections of the bundle $C_{K}=J^{1} L X / G L(4, \mathbb{R})$. Pseudo-Riemannian metrics on $X$ are sections of the quotient bundle $\Sigma=L X / O(1,3)$. The total configuration space of gravitation theory $\Sigma \times C_{K}$, coordinated by $\left(x^{\lambda}, \sigma^{\alpha \beta}, k_{\mu}{ }^{\alpha} \beta\right)$, admits the functorial lift

$$
\begin{aligned}
\tau+ & \left(\sigma^{v \beta} \partial_{v} \tau^{\alpha}+\sigma^{\alpha v} \partial_{\nu} \tau^{\beta}\right) \frac{\partial}{\partial \sigma^{\alpha \beta}} \\
& +\left(\partial_{v} \tau^{\alpha} k_{\mu}{ }^{v}{ }_{\beta}-\partial_{\beta} \tau^{v} k_{\mu}{ }^{\alpha}{ }_{v}-\partial_{\mu} \tau^{v} k_{v}{ }^{\alpha}{ }_{\beta}+\partial_{\mu \beta} \tau^{\alpha}\right) \frac{\partial}{\partial k_{\mu}{ }^{\alpha}{ }_{\beta}}
\end{aligned}
$$

of vector fields $\tau$ on $X$ [10]. Let a gravitation Lagrangian $L_{M A}$ on the jet manifold $J^{1}\left(\Sigma \times C_{K}\right)$ be invariant under general covariant transformations. Then the variational derivatives $\mathscr{E}_{\alpha \beta}, \mathscr{E}^{\mu}{ }_{\alpha} \beta$ of this Lagrangian obey the irreducible NI

$$
\begin{aligned}
& -\left(\sigma_{\lambda}^{\alpha \beta}+2 \sigma_{v}^{v \beta} \delta_{\lambda}^{\alpha}\right) \mathscr{E}_{\alpha \beta}-2 \sigma^{v \beta} d_{v} \mathscr{E}_{\lambda \beta}+\left(-k_{\lambda \mu}{ }^{\alpha}{ }_{\beta}-k_{v \mu}{ }^{v}{ }_{\beta} \delta_{\lambda}^{\alpha}+k_{\beta \mu}{ }^{\alpha} \lambda\right. \\
& \left.\quad+k_{\mu \lambda}{ }^{\alpha}{ }_{\beta}\right) \mathscr{E}^{\mu}{ }_{\alpha}{ }^{\beta}+\left(-k_{\mu}{ }^{v}{ }_{\beta} \delta_{\lambda}^{\alpha}+k_{\mu}{ }^{\alpha}{ }_{\lambda} \delta_{\beta}^{v}+k_{\lambda}{ }^{\alpha}{ }_{\beta} \delta_{\mu}^{v}\right) d_{\nu} \mathscr{E}^{\mu}{ }_{\alpha}{ }^{\beta}+d_{\mu \beta} \mathscr{E}_{\lambda}{ }_{\lambda}{ }^{\beta}=0 .
\end{aligned}
$$

Taking the vertical part of vector fields $\tilde{\tau}$ and replacing gauge parameters $\tau^{\lambda}$ with ghosts $c^{\lambda}$, we obtain the gauge operator $u_{e}$ and its nilpotent BRST prolongation

$$
\begin{aligned}
u_{E}= & \left(\sigma^{v \beta} c_{v}^{\alpha}+\sigma^{\alpha v} c_{v}^{\beta}-c^{\lambda} \sigma_{\lambda}^{\alpha \beta}\right) \frac{\partial}{\partial \sigma^{\alpha \beta}}+ \\
& \left(c_{v}^{\alpha} k_{\mu}{ }^{v}{ }_{\beta}-c_{\beta}^{v} k_{\mu}{ }^{\alpha}{ }_{v}-c_{\mu}^{v} k_{v}{ }^{\alpha}{ }_{\beta}+c_{\mu \beta}^{\alpha}-c^{\lambda} k_{\lambda \mu}{ }^{\alpha}{ }_{\beta}\right) \frac{\partial}{\partial k_{\mu}{ }^{\alpha}{ }_{\beta}}+c_{\mu}^{\lambda} c^{\mu} \frac{\partial}{\partial c^{\lambda}} .
\end{aligned}
$$


Accordingly, an original gravitation Lagrangian $L_{M A}$ is extended to a proper solution

$$
\begin{aligned}
L_{E}= & L_{M A}+\left(\sigma^{v \beta} c_{v}^{\alpha}+\sigma^{\alpha v} c_{v}^{\beta}-c^{\lambda} \sigma_{\lambda}^{\alpha \beta}\right) \bar{\sigma}_{\alpha \beta} d^{n} x+ \\
& \left(c_{v}^{\alpha} k_{\mu}{ }^{v}{ }_{\beta}-c_{\beta}^{v} k_{\mu}{ }^{\alpha}{ }_{v}-c_{\mu}^{v} k_{v}{ }^{\alpha}{ }_{\beta}+c_{\mu \beta}^{\alpha}-c^{\lambda} k_{\lambda \mu}{ }^{\alpha}{ }_{\beta}\right) \bar{k}^{\mu}{ }_{\alpha}{ }^{\beta} d^{n} x+c_{\mu}^{\lambda} c^{\mu} \bar{c}_{\lambda} d^{n} x
\end{aligned}
$$

of the master equation where $\bar{\sigma}_{\alpha \beta}, \bar{k}^{\mu}{ }_{\alpha}{ }^{\beta}$ and $\bar{c}_{\lambda}$ are the corresponding antifields.

3) Topological BF theory exemplifies an Abelian reducible degenerate Lagrangian theory which satisfies the homology regularity condition [2, 4]. Its dynamic variables are exterior forms $A$ and $B$ of form degree $|A|+|B|=n-1$ on a manifold $X$. They are sections of the bundle $Y=\stackrel{p}{\wedge} T^{*} X \oplus \stackrel{q}{\wedge} T^{*} X, p+q=n-1$, coordinated by $\left(x^{\lambda}, A_{\mu_{1} \ldots \mu_{p}}, B_{v_{1} \ldots v_{q}}\right)$. Without a loss of generality, let $q$ be even and $q \geq p$. The corresponding DGA is $\mathscr{O}_{\infty}^{*} Y$. There are the canonical $p$ - and $q$-forms

$$
A=\frac{1}{p !} A_{\mu_{1} \ldots \mu_{p}} d x^{\mu_{1}} \wedge \cdots \wedge d x^{\mu_{p}}, \quad B=\frac{1}{q !} B_{v_{p+1} \ldots v_{q}} d x^{v_{p+1}} \wedge \cdots \wedge d x^{v_{p}}
$$

on $Y$. A Lagrangian $L_{\mathrm{BF}}=A \wedge d_{H} B$ leads to Euler-Lagrange equations $d_{H} A=0$, $d_{H} B=0$ obeying NI $d_{H} d_{H} A=0, d_{H} d_{H} B=0$. Given the vector bundles

$$
\begin{aligned}
& E_{k}=\stackrel{p-k-1}{\wedge} T^{*} X \underset{X}{\times} \wedge^{q-k-1} T^{*} X, \quad 0 \leq k<p-1, \quad E_{p-1}=\underset{X}{\mathbb{R}} \stackrel{q}{\wedge}^{q-p} T^{*} X, \\
& E_{k}=\stackrel{q-k-1}{\wedge} T^{*} X, \quad p-1<k<q-1, \quad E_{q-1}=X \times \mathbb{R},
\end{aligned}
$$

let us consider the DGA $P_{\infty}^{*}\{q-1\}$ with a local basis

$$
\begin{aligned}
& \left\{A_{\mu_{1} \ldots \mu_{p}}, B_{v_{p+1} \ldots v_{q}}, \varepsilon_{\mu_{2} \ldots \mu_{p}}, \ldots, \varepsilon_{\mu_{p}}, \varepsilon, \xi_{v_{p+2} \ldots v_{q}}, \ldots, \xi_{v_{q}}, \xi\right. \\
& \left.\bar{A}^{\mu_{1} \ldots \mu_{p}}, \bar{B}^{v_{p+1} \ldots v_{q}}, \bar{\varepsilon}^{\mu_{2} \ldots \mu_{p}}, \ldots, \bar{\varepsilon}^{\mu_{p}}, \bar{\varepsilon}, \bar{\xi}^{v_{p+2} \ldots v_{q}}, \ldots, \bar{\xi}^{v_{q}}, \bar{\xi}\right\} .
\end{aligned}
$$

Then the gauge operator $u_{e}(20)$ reads

$$
\begin{aligned}
u_{e}= & d_{\mu_{1}} \varepsilon_{\mu_{2} \ldots \mu_{p}} \frac{\partial}{\partial A_{\mu_{1} \mu_{2} \ldots \mu_{p}}}+d_{v_{p+1}} \xi_{v_{p+2} \ldots v_{q}} \frac{\partial}{\partial B_{v_{p+1} v_{p+2} \ldots v_{q}}} \\
& +\left[d_{\mu_{2}} \varepsilon_{\mu_{3} \ldots \mu_{p}} \frac{\partial}{\partial \varepsilon_{\mu_{2} \mu_{3} \ldots \mu_{p}}}+\cdots+d_{\mu_{p}} \varepsilon \frac{\partial}{\partial \varepsilon^{\mu_{p}}}\right] \\
& +\left[d_{v_{p+2}} \xi_{v_{p+3} \ldots v_{q}} \frac{\partial}{\partial \xi_{v_{p+2} v_{p+3} \ldots v_{q}}}+\cdots+d_{v_{q}} \xi \frac{\partial}{\partial \xi^{v_{q}}}\right] .
\end{aligned}
$$

This operator is obviously nilpotent and, thus, is the BRST operator. Consequently, the proper extension of the Lagrangian $L_{\mathrm{BF}}$ takes the form (44):

$$
\begin{aligned}
L_{E}= & L_{\mathrm{BF}}+\left[\varepsilon_{\mu_{2} \ldots \mu_{p}} d_{\mu_{1}} \bar{A}^{\mu_{1} \mu_{2} \ldots \mu_{p}}+\varepsilon_{\mu_{3} \ldots \mu_{p}} d_{\mu_{2}} \bar{\varepsilon}^{\mu_{2} \mu_{3} \ldots \mu_{p}}+\cdots+\varepsilon d_{\mu_{p}} \bar{\varepsilon}^{\mu_{p}}\right] d^{n} x+ \\
& {\left[\xi_{v_{p+2} \ldots v_{q}} d_{v_{p+1}} \bar{A}^{v_{p+1} v_{p+2} \ldots v_{q}}+\xi_{v_{p+3} \ldots v_{q}} d_{v_{p+2}} \bar{\varepsilon}^{v_{p+2} v_{p+3} \ldots v_{q}}+\cdots+\xi d_{v_{q}} \bar{\varepsilon}^{v_{q}}\right] d^{n} x . }
\end{aligned}
$$




\section{References}

[1] Barnich, G., Brandt, F. and Henneaux, M.: Local BRST cohomology in gauge theories, Phys. Rep. 338 439-569 (2000)

[2] Bashkirov, D., Giachetta, G., Mangiarotti, L. and Sardanashvily, G.: Noether's second theorem in a general setting. Reducible gauge theories, J. Phys. A 38 5329-5344 (2005)

[3] Bashkirov, D., Giachetta, G., Mangiarotti, L. and Sardanashvily, G.: Noether's second theorem for BRST symmetries, J. Math. Phys. 46053517 (2005)

[4] Bashkirov, D., Giachetta, G., Mangiarotti, L. and Sardanashvily, G.: The antifield Koszul-Tate complex of reducible Noether identities, J. Math. Phys. 46 103513 (2005)

[5] Batalin, I. and Vilkovisky, G.: Closure of the gauge algebra, generalized Lie algebra equations and Feynman rules, Nucl. Phys. B234 106-124 (1984)

[6] Fisch, J. and Henneaux, M.: Homological perturbation theory and algebraic structure of the antifield-antibracket formalism for gauge theories, Commun. Math. Phys. 128 627-640 (1990)

[7] Fulp, R., Lada, T. and Stasheff, J.: Noether variational Theorem II and the BV formalism, Rend. Circ. Mat. Palermo (2) Suppl. No. 71 115-126 (2003)

[8] Giachetta, G., Mangiarotti, L. and Sardanashvily, G.: Lagrangian supersymmetries depending on derivatives. Global analysis and cohomology, Commun. Math. Phys. 259 103-128 (2005)

[9] Gomis, J., París, J. and Samuel, S.: Antibracket, antifields and gauge theory quantization, Phys. Rep. 295 1-145 (1995)

[10] Mangiarotti, L. and Sardanashvily, G.: Connections in Classical and Quantum Field Theory, World Scientific, Singapore (2000)

[11] Sardanashvily, G.: Graded infinite order jet manifolds, Int. J. Geom. Methods Mod. Phys. 4 N8 (2007) 